\section{Volumetric Method for the Determination of Zirconium}

KumrNs ${ }^{1}$ observed that mandelic acid separates zirconium quantitatively from a large number of elements, and its use as a specific reagent for zirconium is being extended in analytical practice ${ }^{2-4}$, in preference to the time-honoured methods of affecting the separation as phosphate or basic selenite ${ }^{5}$. In a series of papers, Klingenberg and his colleagues ${ }^{6,7}$ have investigated the possibility of using $\alpha$-hydroxy carboxylic acids as specific reagents for zirconium determinations and have shown that a direct weighing of $p$-chloro- and $p$-bromo-mandelates of zirconium is a satisfactory procedure for the gravimetric determination of the metal. The halo-derivatives of mandelic acid exert a large weighting effect on the zirconium ion and further, unlike the zirconium mandelate, these are not water-soluble. Hahn ${ }^{8}$ has further explored the use of $p$-bromo-mandelic acid for the determination of the zirconium - hafnium ratio by a computation from the combined weights of the $p$-bromo-mandelate of zirconium and hafnium and the weight of the oxides obtained therefrom by ignition. Jonckers ${ }^{9}$ has studied the reactivity of groups in relation to their application to inorganic analysis.

We have been engaged in a study of the cerimetric oxidation of organic acids and have found that mandelic acid can be quantitatively oxidized by ceric sulphate ${ }^{10.11}$ : the volume of ceric salt consumed can be used as a measure of the mandelic acid content. It was also noted that the same reaction can be extended to $p$-chloro- and $p$-bromo-mandelic acids.

In the present investigation we have explored the possibility of developing a volumetric method for the determination of zirconium by taking advantage of the above reaction.

Zirconium tetra $p$-chloro-mandelate was precipitated from a known volume of the zirconium solution by following the method of Klingenberg, filtered through a sintered-glass funnel of medium porosity, washed, dried and weighed. The halomandelate was dissolved from the crucible by a hot solution of ammonia, the solution acidified and boiled for fifteen minutes with a known excess of standard ceric sulphate solution. The excess was titrated back with standard ferrous sulphate solution using ferroin as internal indicator. The reaction may be represented as follows :

$$
\begin{aligned}
& \mathrm{Zr}\left(\mathrm{Cl} \cdot \mathrm{C}_{6} \mathrm{H}_{4} \mathrm{CH}(\mathrm{OH}) \cdot \mathrm{COO}\right)_{4}+8 \mathrm{Ce}\left(\mathrm{SO}_{4}\right)_{2} \rightarrow \\
& \mathrm{Zr}\left(\mathrm{SO}_{4}\right)_{2}+4 \mathrm{Ce}_{2}\left(\mathrm{SO}_{4}\right)_{3}+4 \mathrm{Cl}_{6} \cdot \mathrm{C}_{6} \mathrm{H}_{4} \mathrm{CHO}+ \\
& 4 \mathrm{CO}_{2}+2 \mathrm{H}_{2} \mathrm{SO}_{4} \text {; }
\end{aligned}
$$

or 1 gm.mol. of zirconium tetra $p$-chloro-mandelate reacts with 8 gm.mol. of ceric sulphate. Similar reactions apply to the zirconium tetra $p$-bromomandelate, and the same procedure was followed for precipitating, dissolving and titrating the zirconium salt.

In Table 1 are given typical figures obtained on $2 \mathrm{ml}$. each of a sulphuric acid solution of zirconium oxide.

It may be seen from the above that the cerimetric oxidation method is applicable to the volumetric determination of zirconium, and the results obtained are reproducible with a great degree of accuracy. The method is being extended to the determination of zirconium in ores and steels. It gives promise of application to even smaller quantities, and full details will be published elsewhere.
Table 1

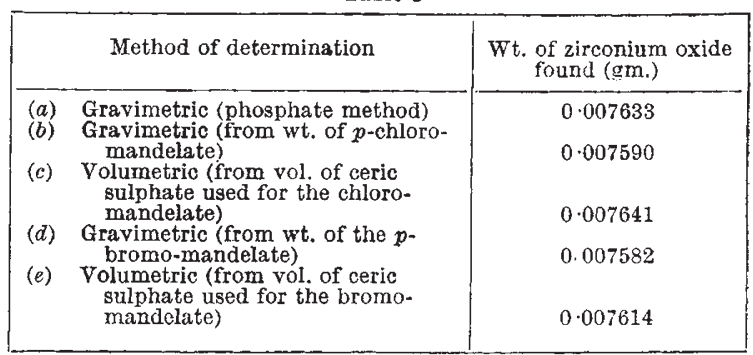

Our thanks are due to Dr. R. C. Shah, of the National Chemical Laboratory of India, Poona, who supplied the halo-mandelic acids, and to Sir K. S. Krishnan, director of the National Physical Laboratory of India, for his kind interest in the work and his permission to publish this communication.

National Physical Laboratory of India,

Mulk RaJ Verma

Sukh Deo Paur

Hillside Road,

New Delhi.

Jan. 30.

1 Kumins, C. A., Anal. Chem., 19, 376 (1947).

2 Gavioli, G., and Traldi, E., La Metallurgia, 42, 179 (1950)

3 Mills, E. C., and Hermon, S. E., Analyst, 78, 256 (1953).

“ IIahn, R. B., Anal. Chem., 2!, 1579 (1949).

5 Hillebrand, W. F., and Lundell, C. E. F., "Applied Inorganic Analysis", 446 et seq. (John Wiley, New York, 1948).

'Klingenberg, J. J., and Oesper, R. E., Anal. Chem., 2!, 1509 (1949).

' Klingenberg, J. J., and Papucci, R. A., Anul. Chem., 24, 1861 (1952).

${ }^{8}$ Hahn, R. B., Anal. Chem., 23, 1259 (1951).

' Jonckers, M. D. E., Chim. Anal., 32, 207 (1950). Cf. Chem. Abs., 45, 61 (1951).

${ }^{10}$ Verma, M. R., and Paul, S. D., J. Sci. and Indust. Res., 12, B, 178 (1953).

${ }^{11}$ Verma, M. R., and Paul, S. D., J. Sci. and Indust. Res. (in the press),

\section{Structure of Pristimerin}

Bhatnagar and Divekar ${ }^{1}$ described the isolation of an antibiotic, an orange-coloured pigment which they called 'pristimerin', from Pristimerae indica and $P$. grahami (Celastraceae). They found that this compound is particularly active against the viridans group of streptococci. We have been studying the constitution of this complex molecule.

Pristimerin crystallizes in orange needles of melting point $218-219^{\circ}$ from petroleum ether - benzene. On the basis of analytical results and molecular weight: (429) determined by X-rays (private communication from Prof. J. D. Bernal to Col. S. S. Bhatnagar), it has been assigned a molecular formula $\mathrm{C}_{28} \mathrm{H}_{36-38} \mathrm{O}_{4}$. The physical and chemical properties and infra-red and ultra-violet spectroscopic evidence show it to be a $\beta$-quinone derivative. It contains one methoxy group and gives a negative Zerewitinoff test for active hydrogen. The latter observation finds con. firmation in the infra-red absorption spectrum, in which the - $\mathrm{OH}$ band is absent. Reductively acetylated or methylated pristimerin (melting points $246^{\circ}$ and $186^{\circ} \mathrm{C}$. respectively) shows ultra-violet absorption suggestive of an acetoxylated or methoxylated benzene ring system, thus indicating that pristimerin may be a derivative of $\beta$-benzoquinone and not of naphthaquinone, nor of a quinone containing fused-ring systems of more than two aromatic rings.

Methylated pristimerin contains three methoxy groups and one double bond, and forms 2-4 dinitrophenylhydrazone, showing that the fourth oxygen in 